\title{
Testosterone replacement therapy of opioid-induced male hypogonadism improved body composition but not pain perception: a double-blind, randomized, and placebo-controlled trial
}

\section{Dorte Glintborg ${ }^{1,2}$, Henrik Bjarke Vaegter ${ }^{3}$, Louise Lehmann Christensen', Emma Bendix', Thomas Graven-Nielsen ${ }^{4}$, Per Grünwald Andersen ${ }^{3}$ and Marianne Andersen ${ }^{1}$}

${ }^{1}$ Department of Endocrinology, Odense University Hospital, Odense, Denmark, ${ }^{2}$ OPEN, Open Patient data Explorative Network, Odense University Hospital, Region of Southern Denmark, Odense, Denmark, ${ }^{3}$ Unit of Pain Research, Department of Anesthesiology, Odense University Hospital, Odense, Denmark, and ${ }^{4}$ Center for Neuroplasticity and Pain (CNAP), SMI, Department of Health Science and Technology, Faculty of Medicine, Aalborg University, Aalborg, Denmark
Correspondence should be addressed to D Glintborg

Email

dorte.glintborg@rsyd.dk

\begin{abstract}
Background: Hypogonadism is prevalent during opioid treatment, but the effect of testosterone replacement treatment (TRT) on body composition, pain perception, and adrenal function is unclear.

Purpose: To measure changes in body composition, pain perception, quality of life, and adrenal function after TRT or placebo in opioid-treated men with chronic non-malignant pain.

Methods: Double-blind, placebo-controlled study in 41 men (>18 years) with total testosterone $<12 \mathrm{nmol} / \mathrm{L}$ were randomized to 24 weeks TRT (Testosterone undecanoate injection three times/6 months, $n=20$ ) or placebo (placeboinjections, $n=21$ ).

Outcomes: Body composition (lean body mass and fat mass assessed by DXA), clinical pain intensity (numerical rating scale), and experimental pain perception (quantitative sensory assessment), quality of life (SF36), and adrenocorticotrophic hormone (ACTH) test. Data were presented as median (quartiles). Mann-Whitney tests were performed on delta values (24-0 weeks) between TRT and placebo.

Results: The median age was 55 years $(46 ; 59)$ and total testosterone before intervention was $6.8(5.0 ; 9.3) \mathrm{nmol} / \mathrm{L}$. TRT was associated with change of testosterone levels: $12.3(7.0 ; 19.9) \mathrm{nmol} / \mathrm{L}(P<0.001$ vs placebo), increased lean body mass: $3.6(2.3 ; 5.0) \mathrm{kg}$ vs $0.1 \mathrm{~kg}(-2.1 ; 1.5)$ during TRT vs placebo and decreased total fat mass: $-1.2(-3.1 ; 0.7) \mathrm{kg}$ vs $1.2 \mathrm{~kg}(-0.9 ; 2.5) \mathrm{kg}$, both $P<0.003$. Changed pain perception, SF36, and ACTH-stimulated cortisol levels were nonsignificantly changed during TRT compared with placebo.

Conclusions: Six months of TRT improved body composition in men with opioid-induced hypogonadism without significant changes in outcomes of pain perception, quality of life, or adrenal function.
\end{abstract}

\section{Introduction}

Opioid medications are widely used to manage chronic non-cancer pain. Opioids are known to suppress pituitary (c) 2020 European Society of Endocrinology Printed in Great Britain function by inhibition of the relevant hypothalamic releasing factors via opioid receptors, which are widely distributed throughout the CNS (1). One of the most well described hormonal side effects of opioid treatment is male 
hypogonadism. Treatment with opioids increases the risk of secondary hypogonadism, characterized by low serum levels of testosterone and low levels of luteinizing hormone and follicle-stimulating hormone (2). Recent reviews estimate that the risk of hypogonadism ranges from 17 to $89 \%$ in men treated with opioids (3). The risk magnitude of male hypogonadism depends on treatment duration, dosage of opioids, and the applied cut-off level for total testosterone to define hypogonadism (3). Testosterone replacement therapy (TRT) is indicated in men with severe hypogonadism, whereas the indication for TRT in opioidinduced relative hypogonadism in men is debated.

The potential effect of TRT on pain perception remains to be investigated. In animal studies, the opioid and gonadal systems interact and regulate sensitivity to nociceptive stimuli (3). In castrated male rodents, testosterone administration improves pain tolerance to mechanical and thermal nociceptive stimuli (3). Few studies have investigated the effect of TRT on pain outcomes and quality of life in opioid-treated men with relative hypogonadism. A recent meta-analysis (4) found one eligible randomized controlled trial $(n=84$, study duration 3 months) (5) and four observational studies (total $n=157$ ) (4). The authors concluded that very lowquality evidence supported that TRT was associated with improved pain and emotional functioning, whereas TRT did not affect physical functioning, role functioning, or social functioning (4). Furthermore, sexual function is impaired during opioid treatment (6) and data regarding sexual function during TRT in these men are sparse (5). Obesity is inversely associated with testosterone levels (7). Lean body mass is positively associated with higher endogenous pain modulation (8). TRT increases lean body mass and decreases abdominal s.c. adipose tissue $(9,10$, 11). In theory, disability may be linked to inactivity and low lean body mass and therefore, presence of chronic pain could increase the effect of TRT on lean body mass in opioid-treated men compared to other study populations. Whether a positive effect of TRT on pain perception is mediated via improved body composition with higher lean body mass is undetermined.

Secondary adrenal insufficiency is a less described side effect in opioid-treated individuals (12). The prevalence of insufficient adrenal response is estimated to range between 9 and $29 \%$ in men and women treated with long-term opioids $(1,12)$. Adrenal insufficiency will have an adverse effect on for example inflammatory disease, joint, and muscle pain (1). Furthermore, severe adrenal insufficiency should be treated according to available guidelines. Previous studies have used a wide range of diagnostic criteria for adrenal insufficiency (12) and more data are needed regarding risk and possible predictors of adrenal insufficiency in patients treated with opioids. BMI is inversely related to cortisol responsivity $(13,14)$, but whether improved body composition during TRT could affect cortisol levels during the ACTH test remains to be tested.

The aim of the present study was to investigate the effects of TRT on body composition, pain perception, pain sensitivity, adrenal cortisol response, and quality of life in opioid-treated men with relative hypogonadism.

\section{Methods}

\section{Study design}

The study was conducted during from August 2016 to August 2019 as a single center, randomized, placebocontrolled, double-blind study. The study outcomes were evaluated at baseline and after 6 month's (24 weeks) intervention. All participants gave written informed consent. The study was approved by The Regional Scientific Ethical Committees for Southern Denmark (S-20150004) and the Danish Health and Medicines Agency. Monitoring was performed according to good clinical practice (GCP) by the GCP unit at Odense University hospital. The trial was declared in www.clinicaltrials.gov (NCT02433730). The study was planned and approved by Danish authorities in May 2015. Participants were recruited from August 2016 and onward. The authors confirm that all ongoing and related trials for this drug/intervention are registered. Study data were collected and managed using REDCap electronic data capture tools (15) hosted at University of Southern Denmark. The study design is presented in the appendix according to the Consort checklist.

\section{Participants}

The study participants were men aged $>18$ years with total testosterone (T-testosterone) $<12 \mathrm{nmol} / \mathrm{L}$ and treated with opioids for non-malignant pain disease. Inclusion criteria were at least two measures of T-testosterone $<12$ $\mathrm{nmol} / \mathrm{L}$ (measured between $0800 \mathrm{~h}$ and $1000 \mathrm{~h}$ in the morning), treatment with opioids for at least 3 months at a dosage corresponding to at least $50 \mathrm{mg}$ morphine/day (for conversion to morphine equivalent doses, we applied https://www.oregonpainguidance.org/opioidmedcalcul ator/), and LH and prolactin within reference interval. Exclusion criteria were those who wished to conceive 
during the trial period, hematocrit $>53 \%$, previous or ongoing malignant disease, prostate specific antigen (PSA) $>3 \mathrm{ng} / \mathrm{dL}$, untreated ischemic heart or respiratory disease, alcohol or drug abuse, abnormal routine blood samples (TSH, ionized calcium, hemoglobin, liver, and kidney function), treatment with $5 \alpha$-reductase inhibitors and oral glucocorticoid steroids, and previous or current testosterone treatment.

\section{Randomization}

Randomization numbers were assigned to the participants in order of enrollment into the study. The randomization list, medicine labeling, and randomization- and codebreak envelopes were generated by the pharmacy at Odense University Hospital to ensure double blinding.

\section{Interventions}

Participants were randomly assigned to receive testosterone (TRT, testosterone undecanoate (Nebido) $1000 \mathrm{mg}$ ) or placebo injections. The pharmacy at Odense University Hospital handled packaging of medicine for each study participant. Patients then received medicine according to randomization number. Placebo injections were prepared by the pharmaceutical company to ensure identical packaging of TRT and placebo. The study participants received injections at time of randomization and at 6 and 18 weeks.

\section{Outcomes and assessments}

Participants underwent clinical examination, dual X-ray Absorptiometry (DXA) scan, assessment of clinical and experimental pain, fasting blood samples, ACTH test and OGTT, and answered questionnaires by time of study inclusion and the examination program was repeated after 24-weeks intervention.

\section{Body composition measures}

Clinical examination included height, weight, and waist circumference.

\section{DXA}

Trunk fat mass, total fat mass, fat percentage, and lean body mass were measured by DXA using a Hologic Discovery device (Waltham, MA, USA). The CV was $0.8 \%$ for total fat mass and $0.6 \%$ for lean body mass.

\section{Assessment of clinical pain parameters}

Data on clinical pain manifestations were collected by the electronic software system PainData used in the Pain Center at the Odense University Hospital in Denmark. Data was collected on pain duration and intensity of clinical peak pain and clinical average pain on a 0-10 numerical rating scale (NRS), with 0 defined as 'no pain' and 10 'as worst imaginable pain' during the previous $24 \mathrm{~h}$. Absolute difference in NRS pain intensity was calculated as pain intensity at baseline minus pain intensity at 6 months follow-up with positive numbers indicating a reduction in pain intensity at follow-up.

\section{Assessment of experimental pain sensitivity}

Participants underwent assessments of pressure pain thresholds (PPTs and cPPT) and pressure pain tolerance (PTT), a protocol for temporal summation of pressure pain (TSP), a protocol to determine pain wind-up ratio (WUR), a protocol for conditioned pain modulation (CPM), as well as assessment of heat pain thresholds (HPT) and cold pain thresholds (CPT). All pain sensitivity assessments were performed with the participant seated on a plinth with both arms resting on the thighs. The assessment lasted $30 \mathrm{~min}$ and was performed by the same experienced assessor (HBV). Pain assessment was undertaken in the same order for all participants (PPTs, cPPT, PTT, TSP, CPM, WUR, HPT, and CPT). Details regarding experimental pain measurement are presented in the appendix (see section on supplementary materials given at the end of the article).

\section{Questionnaires}

The Short-Form 36 (SF-36) is a generic measure of health status comprising 36 items related to eight dimensions: physical functioning for the limitation in performing all physical activities, role physical for problems with work or other daily activities, bodily pain, general health, vitality, social functioning, role emotional, and mental health (16). QualityMetric's Scoring Solutions and license agreements were obtained from Qualitymetric.com.

\section{Sexual function}

Participants fulfilled a Danish questionnaire regarding sexual function (17). The questionnaire was developed and validated by Søren Buus Jensen (17) and included questions regarding partner status, sexual activity, 
and erectile dysfunction. Erectile dysfunction was defined to be present if the patient agreed to one the following statements regarding sexual intercourse and/or masturbation: I can get erection, but it disappears during intercourse/masturbation; I often have problems getting erection; and it is not possible for me to get erection.

We had information regarding prescription of phosphodiesterase type 5 (PDE5)-inhibitors, but we did not have information regarding actual use. Therefore, use of PDE5-inhibitors was not included in results.

\section{Oral glucose tolerance test (OGTT)}

A 2-h OGTT was performed at $0800 \mathrm{~h}$ after overnight fasting. Blood glucose was measured at 0, 30, 60, and 120 min after oral ingestion of a glucose load containing the equivalent of $75 \mathrm{~g}$ anhydrous glucose dissolved in water. Area under the curve for glucose was calculated applying the trapezium rule.

\section{Adrenocorticotrophic hormone (ACTH) test}

The ACTH test was standardized to be performed after the OGTT. An i.v. bolus of $0.25 \mathrm{mg}$ Synachten (Novartis Healthcare) was administered and cortisol levels were measured at 0 and 30 min. Delta cortisol was defined as cortisol $30 \mathrm{~min}$-cortisol $0 \mathrm{~min}$. We defined insufficient response to ACTH test as $30 \mathrm{~min}$ cortisol value $<420 \mathrm{nmol} / \mathrm{L}$ according to the reference interval at our laboratory.

\section{Biochemical analyses}

\section{Testosterone and SHBG}

Testosterone levels were measured in the morning in the fasting state by liquid chromatography tandem mass spectrometry. For testosterone measurements the intra-assay coefficient of variation was $10 \%$ for total testosterone $>0.2 \mathrm{nmol} / \mathrm{L}$ and $30 \%$ in the range between 0.1-0.2 nmol/L. SHBG was measured by autoDELFIA assay, and bioavailable testosterone (BioT) was calculated according to the formulas of Vermeulen (18), http://www. issam.ch/freetesto.htm. During calculations, we used the assumption that albumin concentration in participants was $4.3 \mathrm{~g} / \mathrm{L}$. The normal range and 95\% CI for BioT was $7.3 \mathrm{nmol} / \mathrm{L}(7.0-7.5 \mathrm{nmol} / \mathrm{L})(7)$.

\section{Lipids}

Plasma total cholesterol, high-density lipoprotein cholesterol (HDL), and triglycerides (TG) were analyzed by enzymatic colorimetric reactions (Modular P, Roche), and low density lipoprotein cholesterol (LDL) was calculated using the Friedewald equation. For fasting lipid parameters, reference intervals were as follows: total cholesterol: 3.6-6.8 mmol/L, LDL: $1.8-4.5 \mathrm{mmol} / \mathrm{L}, \mathrm{HDL}$ : 0.76-1.68 mmol/L, and TG: 0.47-2.31 mmol/L.

Serum cortisol was measured using an automated solid-phase, competitive chemiluminescent enzyme immunoassay using an Immulite 2000 XPi analyzer, Siemens Healthineers. At cortisol level $290 \mathrm{mmol} / \mathrm{L}$, the precision was $\mathrm{CV} 7.7 \%$ (s.D. 22.4) and at cortisol 587 $\mathrm{nmol} / \mathrm{L}$, the $\mathrm{CV}$ was $6.4 \%$ (s.D. 37.8).

Blood glucose was measured with a Hemocue device (Hemocue, Ängelholm, Sweden), which converted blood glucose concentrations to equivalent plasma glucose concentrations (19). The meter was checked with a control cuvette every day and a hemolysate every 1-2 weeks.

\section{Statistical analysis}

By the time of study planning, no study had investigated the effect of TRT on pain perception. Therefore, the sample size of the study was determined by the effect of TRT on lean body mass based on a meta-analysis on testosterone therapy in aging men by Isidori (20). If the study had sufficient power to detect significant changes in lean body mass, we hypothesised that the study would be sufficiently powered to detect clinically relevant changes in pain perception. The inclusion of study participants and number of drop outs is presented in the attached Consort flow diagram (Fig. 1).

Pre-treatment differences between participants in the testosterone and placebo group were tested using MannWhitney $U$ tests. The dataset was of limited size and, therefore, the effects of TRT and placebo were analyzed by comparing delta $(\Delta)$ values of hormonal and metabolic variables using Mann-Whitney test as described by Altman (21). $\Delta$-values on clinical and biochemical markers were calculated as post-treatment level minus pretreatment level. $\Delta$-Pain sensation measures were correlated with $\Delta$-values of total testosterone and body composition using Spearman non-parametric correlation tests.

All statistics were performed using SPSS 17.0 (SPSS Inc) for calculations, and $P$-values $<0.05$ were considered significant. Data are given as median and interquartile range.

\section{Results}

\section{Study population $(n=41)$}

Baseline data are shown in Table 1. The two intervention groups (TRT, $n=20$ and placebo, $n=21$ ) were comparable 


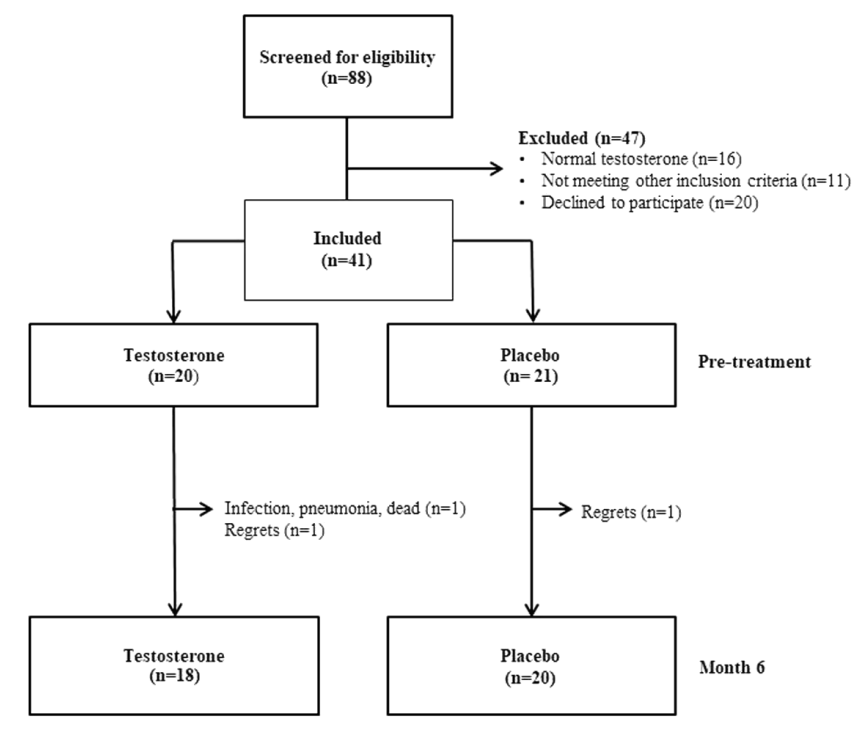

Figure 1

at baseline regarding all clinical and biochemical study outcomes. At baseline, insufficient ACTH test was observed in six men (16\%, three placebo, three TRT). In participants with insufficient cortisol response to the ACTH test, unstimulated cortisol levels ranged from 86 to $192 \mathrm{nmol} / \mathrm{L}$ and stimulated cortisol levels ranged from 372 to $419 \mathrm{nmol} / \mathrm{L}$. The median morphine equivalent dosage (ME) was $163 \mathrm{mg} /$ day (range 86-192 mg/day) in participants with insufficient cortisol response compared
TRT improved SF36 physical component score (PCS), whereas the remaining dimensions of SF36 and visual analog (VAS) scores were unchanged. Changes in experimental pain sensitivity outcomes were not significantly different between TRT and placebo (Table 2).

\section{Bivariate associations between $\Delta$-pain and $\Delta$-body composition and $\Delta$-testosterone levels during TRT}

$\Delta$-peak NRS pain scores showed an inverse association with $\Delta$-T-testosterone $(r=0.50, P=0.04)$ and $\Delta$-Bio $\mathrm{T}(r=0.55$, $P=0.02)$, suggesting that higher change in T-testosterone and Bio $\mathrm{T}$ was associated with larger reductions in peak pain intensity in the TRT group. $\Delta$-pain measures were not significantly associated with $\Delta$-lean body mass or $\Delta$-fat mass (data not shown).

\section{Sexual function}

\section{Baseline} to median morphine equivalent dosage of $90 \mathrm{mg} /$ day (range 45-360 mg/day) in participants with sufficient cortisol response ( $P=0.22$ between groups).

\section{TRT vS placebo treatment}

TRT was associated with higher testosterone levels (total and free testosterone), improved body composition (increased lean body mass and decreased BMI, fat trunk, total fat mass, and fat percentage), unchanged lipids (T-cholesterol, LDL, TG, and HDL (HDL not shown)), and unchanged glucose levels (fasting blood glucose, 2-h blood glucose, and AUC glucose during OGTT) (Table 1).

Changes in cortisol levels during the ACTH test (cortisol 30 min and delta cortisol) were comparable between TRT and placebo. The number of participants with insufficient cortisol response during ACTH test was unchanged during medical intervention (three participants in TRT and three participants in placebo) and no participants changed response pattern from sufficient to insufficient response or vice versa during the treatment period.
A total of 23/36 men participating in the study were married (16/19 TRT vs $12 / 17$ placebo, $P=0.43)$ with median marriage duration of 16 years $(6 ; 28)$ and father of two $(2 ; 3)$ children. At baseline, erectile dysfunction was present in $23 / 35$ (65\%) men $(12 / 18$ vs $11 / 17, P=0.9)$ with no ability to obtain erection in $13 / 35$ men $(8 / 18$ vs $5 / 17, P=0.6)$, whereas $12 / 35(6 / 18$ vs $6 / 17, P=0.56)$ had no erection problems. At baseline, $27 / 35$ (13/18 vs 14/17, $P=0.34)$ men had coitus at intervals longer than 1 month with average duration since last coitus of $150(10 ; 1662)$ days $(150(10 ; 2007)$ vs $257(13 ; 1095))$ days, $P=0.7)$.

\section{TRT vs placebo}

Partner status was unchanged in all men during the study. At 6 months, erectile dysfunction was present in $14 / 32$ men $(4 / 16$ vs $10 / 16, P=0.03)$ with no ability to obtain erection in $10 / 32(3 / 16$ vs $7 / 16, P=0.10)$, whereas $18 / 32(12 / 16$ vs $6 / 16, P=0.03)$ reported no erection problems. At 6 months, $20 / 31$ (9/16 vs $11 / 15, P=0.32)$ had coitus at intervals longer than a month with average duration since last coitus of $18(6 ; 1825)$ days in TRT vs $365(30 ; 1450)$ days in the placebo group $(P=0.04 \Delta$-TRT vs $\Delta$-placebo).

\section{Discussion}

In the present study, body composition improved during 6 months randomized intervention with TRT without 


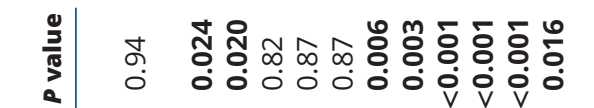

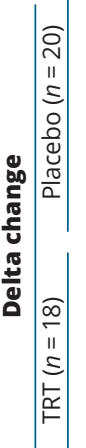

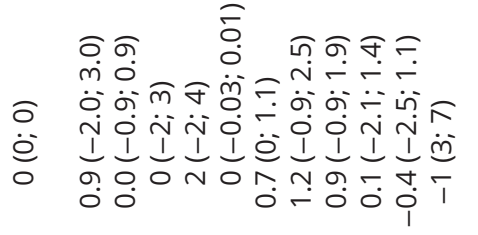

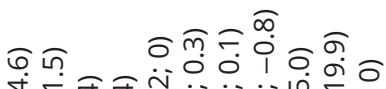

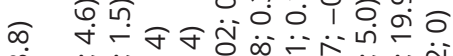

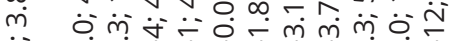

Oे

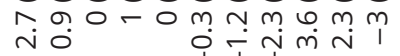

के $\bar{m} \widehat{\phi} \bar{\omega} \bar{\omega}$

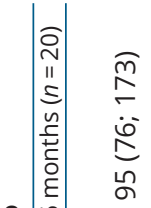

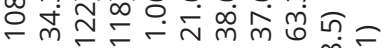

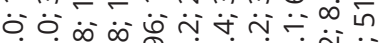

o

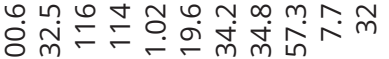

สิ่

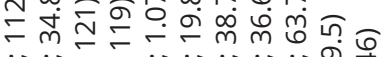
ớ क्

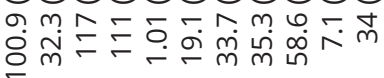

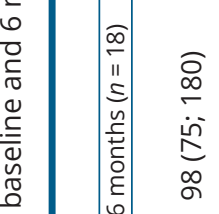

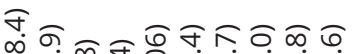

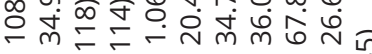

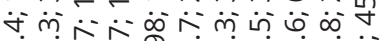

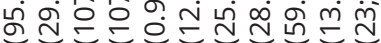

ᄂ? $r .=5 \infty$ o.

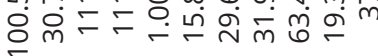

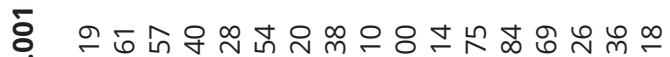

คั ติำ

$\stackrel{\circ}{\circ}$

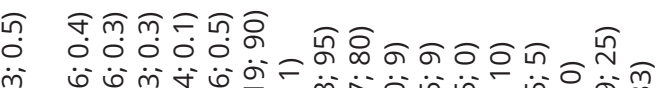

ợ mơ

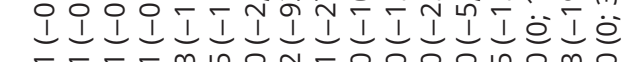

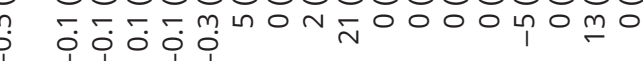

$1 \quad 1,01$

ลू

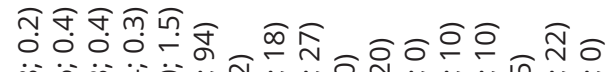

o

IIIIIIIIIIIIIIIIII

ñ.

1
in
on
1
1

कर

$\dot{\infty} \infty \dot{\infty}^{\circ}$

$0 \underset{1}{1}$

$\sqrt{n}$

는

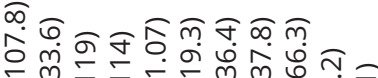

ó

$\infty$ 車

0.

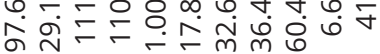

=

oi $\because \ddot{0} \dot{n}$ in

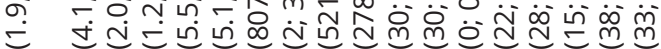

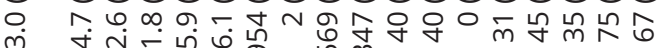

త্ণ

这范

$\stackrel{\sim}{\wedge}$

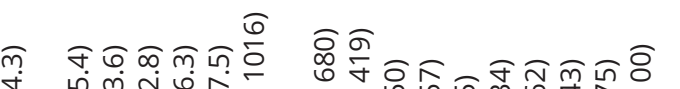

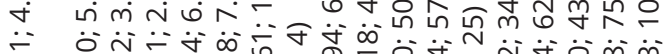

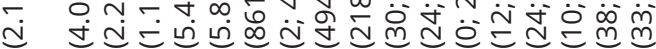

子

₹舀

î임

응

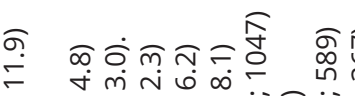

花

- $m$ nom

o

品

อิ

ते $\ddot{\circ}$

$8 \bar{m}$

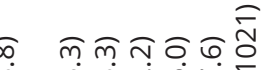

กิธิ

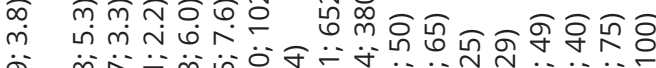

ó 00

들 $=0$

․

冈্

守这

ชั बे

कo

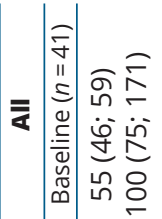

morram

o ळ

0

㐫.

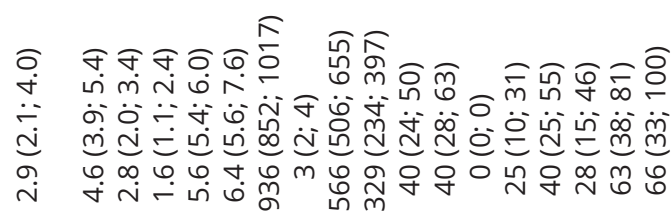

종

를

ชั

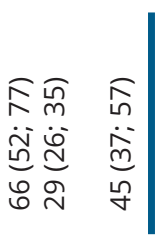

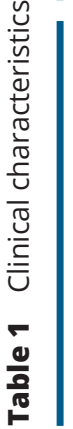
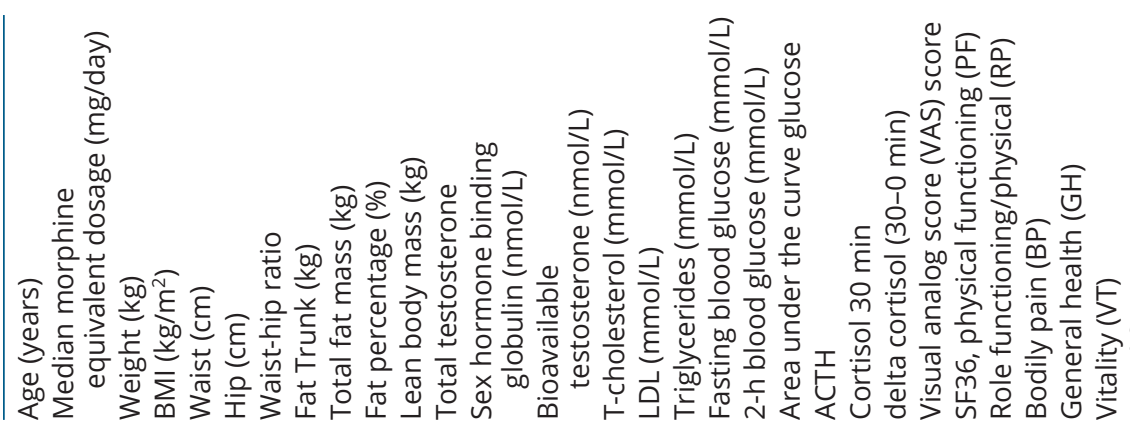

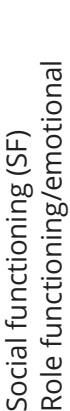

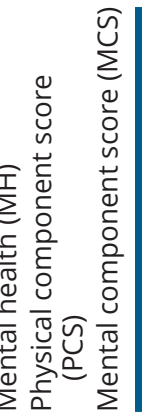


Table 2 Experimental pain sensitivity at baseline and 6 months follow-up in the TRT and placebo groups.

\begin{tabular}{|c|c|c|c|c|c|c|c|}
\hline & \multicolumn{2}{|c|}{ TRT } & \multicolumn{2}{|c|}{ Placebo } & \multicolumn{2}{|c|}{ Delta change } & \multirow[b]{2}{*}{$P$ value } \\
\hline & $\begin{array}{l}\text { Baseline } \\
(n=20)\end{array}$ & $\begin{array}{c}6 \text { months } \\
(n=18)\end{array}$ & $\begin{array}{l}\text { Baseline } \\
(n=21)\end{array}$ & $\begin{array}{c}6 \text { months } \\
(n=20)\end{array}$ & $\operatorname{TRT}(n=18)$ & $\begin{array}{c}\text { Placebo } \\
(n=20)\end{array}$ & \\
\hline $\begin{array}{l}\text { Peak pain } \\
\text { numerical rating } \\
\text { scale }\end{array}$ & $8.0(7.0 ; 8.8)$ & $9.0(7.0 ; 10.0)$ & $7.0(6.0 ; 8.5)$ & $8.0(6.3 ; 8.0)$ & $-0.5(-1.0 ; 0.0)$ & $0.3(-0.8 ; 1.8)$ & 0.13 \\
\hline $\begin{array}{l}\text { Average pain } \\
\text { numerical } \\
\text { rating scale }\end{array}$ & $6.0(4.3 ; 7.0)$ & $6.0(5.0 ; 8.0)$ & $5.0(4.0 ; 6.5)$ & $5.0(3.3 ; 6.0)$ & $-0.2(-0.5 ; 0.0)$ & $0.45(0.0 ; 1.8)$ & 0.18 \\
\hline $\begin{array}{l}\text { Heat pain } \\
\text { threshold }\left({ }^{\circ} \mathrm{C}\right)\end{array}$ & $44.5(40.2 ; 46.5)$ & $43.3(39.2 ; 47.3)$ & $41.1(38.3 ; 45.7)$ & $42.6(39.4 ; 46.5)$ & $-0.3(-2.5 ; 1.8)$ & $0.8(-2.3 ; 3.4)$ & 0.45 \\
\hline $\begin{array}{l}\text { Cold pain } \\
\text { threshold }\left({ }^{\circ} \mathrm{C}\right)\end{array}$ & $11.9(5.1 ; 20.6)$ & $13.8(5.7 ; 20.8)$ & $10.0(5.0 ; 17.9)$ & $12.3(6.6 ; 15.3)$ & $1.7(-2.9 ; 6.3)$ & $0.4(-1.2 ; 2.5)$ & 0.80 \\
\hline $\begin{array}{l}\text { Pressure pain } \\
\text { thresholds (kPa) }\end{array}$ & $372(292 ; 530)$ & $355(211 ; 387)$ & $314(233 ; 443)$ & $310(252 ; 430)$ & $-58(-163 ; 53)$ & $-2(-71 ; 59)$ & 0.25 \\
\hline $\begin{array}{l}\text { Cuff pressure pain } \\
\text { threshold }(\mathrm{kPa})\end{array}$ & $20.5(16.0 ; 28.2)$ & $20.5(15.2 ; 29.1)$ & $22.2(14.0 ; 26.0)$ & $21.4(16.6 ; 25.8)$ & $-1.3(-6.5 ; 4.3)$ & $2.1(-2.3 ; 5.6)$ & 0.12 \\
\hline $\begin{array}{c}\text { Cuff pressure pain } \\
\text { tolerance }(\mathrm{kPa})\end{array}$ & $45.0(30.9 ; 59.7)$ & $42.9(30.5 ; 80.0)$ & $44.0(31.8 ; 52.9)$ & $47.1(30.9 ; 52.9)$ & $-3.6(-14.3 ; 2.6)$ & $0.1(-5.3 ; 4.7)$ & 0.29 \\
\hline $\begin{array}{l}\text { Cuff-induced } \\
\text { temporal } \\
\text { summation of } \\
\text { pressure pain } \\
\text { (ratio) }\end{array}$ & $2.2(1.5 ; 2.6)$ & $1.9(1.4 ; 2.5)$ & $1.9(1.2 ; 2.9)$ & $1.7(1.3 ; 3.5)$ & $0.5(-0.9 ; 1.2)$ & $-0.1(-1.5 ; 1.5)$ & 0.75 \\
\hline Wind-up ratio & $2.0(0.5 ; 3.0)$ & $3.0(1.0 ; 5.3)$ & $1.0(0.0 ; 2.0)$ & $1.5(0.0 ; 2.3)$ & $0.6(-1.0 ; 1.0)$ & $0.9(0.0 ; 2.0)$ & 0.46 \\
\hline $\begin{array}{l}\text { Conditioned pain } \\
\text { modulation }(\mathrm{kPa})\end{array}$ & $6.8(1.0 ; 16.1)$ & $4.2(-1.4 ; 14.3)$ & $7.5(3.8 ; 11.9)$ & $6.9(1.5 ; 10.3)$ & $-2.5(-6.7 ; 5.1)$ & $-1.2(-8.8 ; 5.0)$ & 0.52 \\
\hline
\end{tabular}

Data presented as median (quartiles). $P$-value represents unpaired Mann-Whitney test on changes (delta values) during intervention with testosterone vs placebo.

significant changes in clinical and experimental pain perception and adrenal function in men treated with opioids for non-malignant chronic pain disease. The present study is the first long-term randomized term study that investigated possible associations between improved body composition (higher lean body mass), quality of life, and pain perception during TRT in a male study population treated with opioids.

\section{Body composition}

A median increase in lean body mass of $3.6 \mathrm{~kg}$ was observed during TRT, whereas fat mass decreased with median $1.2 \mathrm{~kg}$. A positive effect of TRT treatment on body composition has previously been reported in other study populations (20) and change in lean body mass was our primary study outcome. According to the meta-analysis by Isidori et al. (20), our power calculation was based on an expected increase in lean body mass of $1.6 \mathrm{~kg}$. The meta-analysis (20) was based on randomized placebocontrolled studies performed in diverse study populations and of different study duration, whereas limited data regarding change in body composition during TRT are available in opioid-induced male hypogonadism
(5). Basaria et al. described on average $1.0 \mathrm{~kg}$ increase in lean body mass during 3 month intervention with transdermal testosterone gel in opioid-treated men (5). Importantly, men included in the study by Basaria et al. (5) were comparable to our study population regarding inclusion criteria (both studies included opioid-treated men with testosterone $<12 \mathrm{nmol} / \mathrm{L}$ ), mean age (49 vs 54 years), mean BMI (32 vs $29 \mathrm{~kg} / \mathrm{m}^{2}$ ), and mean daily opioid dosage (114 vs $100 \mathrm{mg}$ ). The findings by Basaria et al. (5) supported that men with hypogonadism due to opioid treatment had similar improvement in lean body mass during TRT compared to men with hypogonadism due to other diseases (20). Post treatment total testosterone was $27.2 \mathrm{nmol} / \mathrm{L}$ in the study by Basaria et al. (5) compared to $19.3 \mathrm{nmol} / \mathrm{L}$ in the present study, which could support that our finding of higher gain of lean body mass could be explained by longer treatment duration of TRT and not average serum testosterone concentration. We found that fat mass decreased median $1.2 \mathrm{~kg}$ during TRT, which was comparable to results reported by Basaria et al. (average loss in fat mass $0.8 \mathrm{~kg}$ ) (5) and by Isidori et al. (average loss of fat mass $1.6 \mathrm{~kg})(20)$. The present data therefore add to the evidence that body composition is improved during TRT and that the positive effect of TRT on body 
composition is not dependent on patient cohort but is dependent of testosterone dosage and treatment duration.

\section{Pain perception}

Measures of clinical pain intensity, experimental pain sensitivity, and measures of quality of life were unchanged during TRT in the present study, which contrasted our study hypothesis. By time of study planning, animal studies (3) and human uncontrolled studies (4) had shown improvements in pain perception and quality of life during TRT (4). Although the study size was limited, all outcomes of pain sensitivity measures were unchanged during study intervention, which did not support a placebo effect. The SF36 PCS domain was significantly increased during TRT, but all other SF36 domains, numerical rating scale (NRS) of pain, and VAS score were unchanged during TRT, which did not support an overall benefit of TRT on quality of life. It is possible that the significant change in the SF36 domain PCS should be considered to be caused by chance as no other SF36 outcomes showed a trend toward significant changes. At present, the only available placebo-controlled study regarding changes in pain and QoL during TRT is the study by Basaria et al. (5), whereas remaining studies were uncontrolled (4). In contrast to the present study, Basaria et al. reported changes in some but not all pain sensitivity measures with significantly higher changes in pressure pain threshold at the thumb and lower pain intensity after ten pinprick stimulations at the hand after TRT, whereas changes in pressure pain threshold at the trapezius muscle, pain intensity after 1 pinprick stimulation, cold pain tolerance, self-reported clinical pain, and SF-36 outcomes were not significantly different between TRT and placebo (5). As already mentioned, study participants in the study by Basaria et al. (5) were comparable to the present study, but participants obtained higher serum levels of total testosterone during TRT, and the effect of TRT on lean body mass was more modest than in the present study. In accordance, in the present study, the reduction in NRS peak pain was associated with $\Delta$-testosterone, whereas changes in pain measures were not associated with changes in body composition.

The findings by Basaria (5) and the present study supported that possible effects of TRT on pain modulation and Qol were mediated primarily by higher testosterone levels instead of increased lean body mass. Furthermore, it could be important to note that the study duration was 3 months in the study by Basaria et al. (5) in contrast to the study duration of 6 months in the present study. It is possible that a modest positive effect of TRT on pain modulation could wear off during prolonged treatment. In accordance, 6 months androgen deprivation therapy in men with prostate cancer resulted in worsening of depression scores and Qol, whereas pain sensitivity and clinical pain were unchanged (22). Available data do not support any clinically significant effects of long-term TRT on clinical and experimental pain, but future studies should discriminate between short and long-term effects of TRT on pain modulation.

\section{Adrenal insufficiency}

Adrenal insufficiency was found in $16 \%$ of men in the present study, and presence of adrenal insufficiency was associated with higher median morphine equivalent dosage. Interestingly, $30 \mathrm{~min}$ cortisol levels during the ACTH test in men with adrenal insufficiency were in all cases only moderately decreased and we found no participants with severe adrenal insufficiency. Increased risk of adrenal insufficiency in opioid-treated patients have been reported in previous studies $(1,12)$; however, different tests were applied for adrenal function assessment $(1,12)$. The adrenal sensitivity for morphine treatment differs between individuals and could be due to opioid receptor polymorphisms resulting in altered opioid receptor affinity or genetic variations in interleukin b, a stimulator of corticotropin and corticotrophin releasing hormone (CRH) (12). We found no significant change of adrenal activity after TRT, despite significant improvements of body composition. Previous studies found an inverse association between BMI and cortisol responsivity $(13,14)$, but it is possible that the change of body composition in the present study was too modest to affect adrenal function. Furthermore, adrenal drive (23) and CBG levels (24) could decrease after weight loss, which could be an alternate explanation of unchanged levels of total cortisol during TRT. Insufficient adrenal function could affect quality of life and low cortisol levels could have an adverse effect on, for example, inflammatory disease and joint and muscle pain (1). Severe adrenal insufficiency should be treated according to available guidelines, but the possible benefits of treating more mild adrenal insufficiency with hydrocortisone regarding quality of life and pain perception remain to be evaluated in future studies.

\section{Sexual function}

Sexual function was significantly improved during TRT as erectile dysfunction improved and sexual activity 
increased. These data supported the findings by Basaria (5) who reported significantly greater increase in sexual desire during TRT, whereas they found no differences in orgasmic domain and erectile function domain between TRT and placebo. More than 50\% men in the present study reported erectile dysfunction and $37 \%$ of participants were not able to have erection. Impaired sexual function in opioid-treated men is well described (6). Present data support that sexual function is improved but not fully restored during TRT, which could be due to unchanged pain perception during TRT.

\section{Strengths and limitations}

Strengths and limitations may apply in the present study. The trial was double-blind and placebo controlled with only few drop outs. The primary study outcome was lean body mass, whereas the study could be underpowered to detect minor changes in secondary study outcomes such as pain perception. However, the use of multiple standardized assessments for pain, pain threshold, and tolerance are an important strength of the study, as the use of a single assessment can lead to questions of reliability. The study was planned during 2014-2015 and, by this time, the placebo-controlled study by Basaria et al. was not published (5). Therefore, the power calculation could not be based on their results. Our study was sufficiently powered regarding our primary study outcome; however, the study could be underpowered to detect minor changes in pain and quality-of-life outcomes. We did not include information about smoking, alcohol, and other lifestyle factors, which could have changed during study intervention and could have affected our study results. Several study outcomes were addressed and therefore the issue of multiple testing needs to be discussed. We could categorize the study outcomes into a limited number of aspects including body composition (weight, lean body mass, total, and regional fat mass), measures of pain perception and quality of life (objective measurements and questionnaires), and adrenal function (ACTH test). The use of several screening modalities for each study outcome strengthened the study conclusion.

\section{Conclusion}

Six-month TRT improved body composition without significant changes in pain perception, pain sensitivity, and quality of life in men with relative opioid-induced hypogonadism.

\section{Supplementary materials}

This is linked to the online version of the paper at https://doi.org/10.1530/ EJE-19-0979.

\section{Declaration of interest}

The authors declare that there is no conflict of interest that could be perceived as prejudicing the impartiality of this study.

\section{Funding}

The authors thank the Novo Nordisk Foundation and the Institute of Clinical Research - University of Southern Denmark. Bayer provided Nebido ${ }^{\circledR}$ and placebo but was otherwise not involved in the study planning or interpretation of results. TGN is a part of Center for Neuroplasticity and Pain founded by the Danish National Research Foundation (DNRF121).

\section{Acknowledgements}

The authors thank the Novo Nordisk Foundation and the Institute of Clinical Research - University of Southern Denmark. Bayer provided Nebido ${ }^{\circledR}$ and placebo but was otherwise not involved in the study planning or interpretation of results. TGN is a part of Center for Neuroplasticity and Pain founded by the Danish National Research Foundation (DNRF121). OPEN is Open Patient data Explorative Network, Odense University Hospital, Region of Southern Denmark (www.sdu.dk/ki/open).

\section{References}

1 Lamprecht A, Sorbello J, Jang C, Torpy DJ \& Inder WJ. Secondary adrenal insufficiency and pituitary dysfunction in oral/transdermal opioid users with non-cancer pain. European Journal of Endocrinology 2018179 353-362. (https://doi.org/10.1530/EJE-18-0530)

2 Ali K, Raphael J, Khan S, Labib M \& Duarte R. The effects of opioids on the endocrine system: an overview. Postgraduate Medical Journal 2016 92 677-681. (https://doi.org/10.1136/postgradmedj-2016-134299)

3 Coluzzi F, Billeci D, Maggi M \& Corona G. Testosterone deficiency in non-cancer opioid-treated patients. Journal of Endocrinological Investigation 201841 1377-1388. (https://doi.org/10.1007/s40618018-0964-3)

4 AminiLari M, Manjoo P, Craigie S, Couban R, Wang L \& Busse JW. Hormone replacement therapy and opioid tapering for opioidinduced hypogonadism among patients with chronic noncancer pain: a systematic review. Pain Medicine 201820 301-313. (https:// doi.org/10.1093/pm/pny040)

5 Basaria S, Travison TG, Alford D, Knapp PE, Teeter K, Cahalan C, Eder R, Lakshman K, Bachman E, Mensing G et al. Effects of testosterone replacement in men with opioid-induced androgen deficiency: a randomized controlled trial. Pain 2015156 280-288. (https://doi.org/10.1097/01.j.pain.0000460308.86819.aa)

6 Ortman HA \& Siegel JA. The effect of methadone on the hypothalamic pituitary gonadal axis and sexual function: a systematic review. Drug and Alcohol Dependence 2020207107823. (https://doi.org/10.1016/j.drugalcdep.2019.107823)

7 Nielsen TL, Hagen C, Wraae K, Brixen K, Petersen PH, Haug E, Larsen R \& Andersen M. Visceral and subcutaneous adipose tissue assessed by magnetic resonance imaging in relation to circulating androgens, sex hormone-binding globulin, and luteinizing hormone in young men. Journal of Clinical Endocrinology and Metabolism 2007 92 2696-2705. (https://doi.org/10.1210/jc.2006-1847)

8 Stolzman S \& Hoeger Bement M. Lean mass predicts conditioned pain modulation in adolescents across weight status. European Journal of Pain 201620 967-976. (https://doi.org/10.1002/ejp.821) 
9 Frederiksen L, Hojlund K, Hougaard DM, Brixen K \& Andersen M. Testosterone therapy increased muscle mass and lipid oxidation in aging men. Age 201234 145-156. (https://doi.org/10.1007/s11357011-9213-9)

10 Frederiksen L, Hojlund K, Hougaard DM, Mosbech TH, Larsen R, Flyvbjerg A, Frystyk J, Brixen K \& Andersen M. Testosterone therapy decreased subcutaneous fat and adiponectin in ageing men. European Journal of Endocrinology 2012166 469-476. (https://doi.org/10.1530/ EJE-11-0565)

11 Magnussen LV, Glintborg D, Hermann P, Hougaard DM, Hojlund K \& Andersen M. Effect of testosterone on insulin sensitivity, oxidative metabolism and body composition in aging men with type 2 diabetes on metformin monotherapy. Diabetes, Obesity and Metabolism 201618 980-989. (https://doi.org/10.1111/dom.12701)

12 Donegan D \& Bancos I. Opioid-induced adrenal insufficiency. Mayo Clinic Proceedings 201893 937-944. (https://doi.org/10.1016/j. mayocp.2018.04.010)

13 Vajravelu ME, Tobolski J, Burrows E, Chilutti M, Xiao R, Bamba V, Willi S, Palladino A, Burnham JM \& McCormack SE. Peak cortisol response to corticotropin-releasing hormone is associated with age and body size in children referred for clinical testing: a retrospective review. International Journal of Pediatric Endocrinology 2015201522. (https://doi.org/10.1186/s13633-015-0018-y)

14 Komarowska H, Stangierski A, Warmuz-Stangierska I, Lodyga M, Ochmanska K, Wasko R, Wanic-Kossowska M \& Ruchala M. Differences in the psychological and hormonal presentation of lean and obese patients with polycystic ovary syndrome. Neuro Endocrinology Letters 201334 669-674.

15 Harris PA, Taylor R, Thielke R, Payne J, Gonzalez N \& Conde JG. Research Electronic Data Capture (REDCap) - a metadata-driven methodology and workflow process for providing translational research informatics support. Journal of Biomedical Informatics 2009 42 377-381. (https://doi.org/10.1016/j.jbi.2008.08.010)

16 McHorney CA, Ware Jr JE, Lu JF \& Sherbourne CD. The MOS 36-item Short-Form Health Survey (SF-36): III. Tests of data quality, scaling assumptions, and reliability across diverse patient groups. Medical Care 199432 40-66. (https://doi.org/10.1097/00005650-19940100000004)
17 Jensen SB, Hagen C, Froland A \& Pedersen PB. Sexual function and pituitary axis in insulin treated diabetic men. Acta Medica Scandinavica: Supplementum 1979624 65-68. (https://doi. org/10.1111/j.0954-6820.1979.tb00721.x)

18 Vermeulen A, Verdonck L \& Kaufman JM. A critical evaluation of simple methods for the estimation of free testosterone in serum. Journal of Clinical Endocrinology and Metabolism 199984 3666-3672. (https://doi.org/10.1210/jcem.84.10.6079)

19 Ignell C \& Berntorp K. Evaluation of the relationship between capillary and venous plasma glucose concentrations obtained by the HemoCue Glucose 201+ system during an oral glucose tolerance test. Scandinavian Journal of Clinical and Laboratory Investigation 201171 670-675. (https://doi.org/10.3109/00365513.2011.619703)

20 Isidori AM, Giannetta E, Greco EA, Gianfrilli D, Bonifacio V, Isidori A, Lenzi A \& Fabbri A. Effects of testosterone on body composition, bone metabolism and serum lipid profile in middleaged men: a meta-analysis. Clinical Endocrinology 200563 280-293. (https://doi.org/10.1111/j.1365-2265.2005.02339.x)

21 Altman DG. Comparing groups - continuous data. Practical Statistics for Medical Research 19911 199-202.

22 Gagliano-Juca T, Travison TG, Nguyen PL, Kantoff PW, Taplin ME, Kibel AS, Manley R, Hally K, Bearup R, Beleva YM et al. Effects of androgen deprivation therapy on pain perception, quality of life, and depression in men with prostate cancer. Journal of Pain and Symptom Management 201855 307-317.e1. (https://doi.org/10.1016/j. jpainsymman.2017.09.017)

23 Woods CP, Corrigan M, Gathercole L, Taylor A, Hughes B, Gaoatswe G, Manolopoulos K, Hogan AE, O'Connell J, Stewart PM et al. Tissue specific regulation of glucocorticoids in severe obesity and the response to significant weight loss following bariatric surgery (BARICORT). Journal of Clinical Endocrinology and Metabolism 2015 100 1434-1444. (https://doi.org/10.1210/jc.2014-4120)

24 Manco M, Fernandez-Real JM, Valera-Mora ME, Dechaud H, Nanni G, Tondolo V, Calvani M, Castagneto M, Pugeat M \& Mingrone G. Massive weight loss decreases corticosteroid-binding globulin levels and increases free cortisol in healthy obese patients: an adaptive phenomenon? Diabetes Care 200730 1494-1500. (https://doi.org/10.2337/dc06-1353)

Received 4 December 2019

Revised version received 5 March 2020

Accepted 26 March 2020 